\title{
De Cuzco a Berlín en el siglo XIX: el gabinete de curiosidades de María Ana Centeno
}

Zara Ruiz Romero

Universidad Pablo de Olavide (España) 



\title{
De Cuzco a Berlín en el siglo XIX: el gabinete de curiosidades de María Ana Centeno ${ }^{1}$
}

\section{From Cuzco to Berlin in the $19^{\text {th }}$ Century: Maria Ana Centeno's Cabinet of Curiosities}

\author{
Zara Ruiz Romero \\ Universidad Pablo de Olavide (España) \\ zmruirom@gmail.com
}

Fecha de recepción: 30 de diciembre de 2019

Fecha de aceptación: 24 de noviembre de 2020

\begin{abstract}
Resumen
María Ana Centeno desarrolló su gabinete de curiosidades y antigüedades precolombinas en el Perú del siglo XIX, en un momento en que la creación de acervos de este tipo resultaba común entre la élite cuzqueña, cada vez más consciente de la importancia de las culturas precolombinas.

Gran parte de la colección fue vendida por sus herederos al Museo Etnológico de Berlín, en una transacción determinada por el valor en alza de las piezas precolombinas en el mercado, y por el creciente interés de viajeros, científicos y exploradores por lugares como Perú, con manifestaciones culturales dignas de estudiar y coleccionar.

En la presente investigación tratamos la formación de la colección Centeno y su posterior viaje, desde Cuzco a Berlín, relacionando su casuística con el contexto y las circunstancias de la época. Asimismo, tomamos como ejemplo la referida colección para reflexionar sobre la pertenencia del patrimonio, y el lugar en que las piezas precolombinas peruanas deberían resguardarse.
\end{abstract}

Palabras clave: Coleccionismo; María Ana Centeno; Arte precolombino; Perú; Museo Etnológico de Berlín; Restitución cultural.

1 Este artículo ha sido realizado como parte del proyecto de $\mathrm{I}+\mathrm{D}+\mathrm{i}$ "Ruinas, expolios e intervenciones en el patrimonio cultural” (ref. UPO-1264180), financiado por el Fondo Europeo de Desarrollo Regional (FEDER) y la Consejería de Transformación Económica, Industria, Conocimientos y Universidades, de la Junta de Andalucía, en el marco del Programa Operativo FEDER Andalucía 2014-2020 (objetivo específico 1.2.3 "Fomento y generación de conocimiento frontera y de conocimiento orientado a los retos de la sociedad, desarrollo de tecnologías emergentes”, porcentaje de cofinanciación FEDER 80\%). 


\begin{abstract}
María Ana Centeno created her cabinet of curiosities and pre-Columbian antiquities during the nineteenth century. In Peru, at that time, the creation of that kind of collection was common between the elite, who were conscious of the increasing value of the pre-Hispanic cultures and antiquities.

Most of the pieces of the collection were sold by María Ana's sons to the Ethnological Museum of Berlin. It was a transaction determined by the rising value of pre-Columbian pieces on the market, and also by the growing interest of travelers, scientists, and explorers in places like Peru.

In that paper, it is addressed the formation of Centeno's collection, and its move from Cuzco to Berlin; all that having into account the contexts and circumstances of the time. Besides, it is made a reflection on the cultural heritage's owners, and the place where the Peruvian pre-Columbian pieces should be.
\end{abstract}

Keywords: Collecting; María Ana Centeno; Pre-Columbian Art; Peru; Ethnological Museum of Berlin; Cultural Restitution.

El coleccionismo es una de las pasiones más idiosincráticas del ser humano. Se remonta desde fechas más antiguas, a grandes civilizaciones como Grecia y Roma, o influyentes personajes de su tiempo, como pudieron ser los monarcas hispanos Carlos V y Felipe II. El deseo de atesorar objetos, considerados únicos y valiosos, se encuentra íntimamente intrincado con la ostentación de poder o conocimientos; coleccionar es una metáfora de cultura (Vázquez, 2001, p. 6). Así, durante la Edad Moderna, la posesión de una colección ya era sinónimo de buen gusto, fortuna y una destacada posición social, una serie de atribuciones que se extienden prácticamente hasta nuestros días.

Mucho se ha hablado sobre la presencia del componente americano en gabinetes y colecciones europeas desde el contacto con el Nuevo Mundo. En este contexto, los objetos correspondientes a las culturas o pueblos originarios precolombinos tuvieron una valoración cambiante, desde una ambivalencia relacionada con el exotismo, el asombro, e incluso el rechazo durante la Edad Moderna, hasta llegar a su consideración como obras de arte, un proceso que aunque comienza mucho antes, culmina en fechas más recientes del siglo XX.

En este escrito, centramos nuestra atención en el coleccionismo de arte precolombino en el seno de su propio país de procedencia, en un fenómeno directamente relacionado con las tendencias que observamos en Europa y, con posterioridad, en América del Norte, y donde la creación de gabinetes y colecciones al modo ilustrado también resultaba bastante común. Temporalmente, viajamos a los primeros años tras la consecución de la Independencia peruana, cuando las obras prehispánicas comienzan a verse inmersas en un destacable proceso de revalorización, directamente relacionado con su consideración como "tesoros nacionales". Focalizamos además nuestro campo de estudio en una colección particular: la atesorada por María Ana Centeno de Romainville (1816/1817-1874), una cuzqueña que nos ofrece el ejemplo 
perfecto para tratar la consideración de las piezas precolombinas, su creciente presencia en colecciones particulares y de museos, y su consecuente dispersión por el extranjero.

\section{NOTAS BIOGRÁFICAS SOBRE MARÍA ANA GENTENO}

María Ana Centeno (también conocida en la historiografía como Ana María, con el apellido escrito de ambas formas, con c y z inicial ${ }^{2}$ ) (Fig. 1) nació en la ciudad de Cuzco en los albores del siglo XIX, en 1816 o 1817 según la fuente consultada. Llegó al mundo en una época tumultuosa para su familia, la ciudad y el país en su conjunto, pues solo unos pocos años más tarde, en 1821, Perú culminará la conquista de su Independencia.

Desde pequeña "fué considerada siempre como la hija modelo, porque durante su vida dió muestras de conservar ese tierno cariño y profunda veneración, que se debe a los autores de nuestra existencia" (García y García, 1925, p. 252), una serie de cualidades que suelen atribuirse al género femenino, cuyas actividades públicas o intelectuales no impedían que debiera mostrarse virtuosa, sumisa y dedicada a los cuidados de sus familiares o convecinos. María Ana cuidó a su madre, Manuela Sotomayor, de quien poco se sabe y que al parecer estuvo enferma durante prácticamente una década (García y García, 1925, p. 253 y Matto de Turner, 1890, p. 198); e igualmente, vendió sus joyas y empeñó sus cosas para ayudar a su padre, Anselmo Centeno, a cubrir el pago de una multa impuesta por el presidente Agustín Gamarra en 1839 (Matto de Turner, 1890, p. 199).

Su padre -heredero de una importante familia y considerado como uno de los personajes de mayor poder en los inicios del Cuzco republicano-, fue jefe de la Casa de la Moneda y director de la Sociedad de Beneficencia Pública de la ciudad; cargos de tamaña importancia que le llevaron a estar involucrado en procesos políticos (Glave, 2004). Sobre estos últimos, destaca el acaecido con Agustín Gamarra, a quien negó ayuda en un momento de necesidad y cuya venganza precipitó la salida de la familia de la ciudad de Cuzco, tras un segundo enfrentamiento, esta vez con el general Juan Crisóstomo Torrico, y en el que Anselmo Centeno fue avergonzado públicamente (Glave, 2004). Por ello, primero se refugiaron en Arequipa, "huyendo de la mofa en un lugar donde había sido 'principal' sin disputa", y más tarde en Lima (Glave, 2004).

2 En las fuentes más antiguas, Bocetos a lápiz de americanos célebres (1890), o La mujer peruana a través de los siglos (1925), encontramos su nombre como María Ana. Pensamos que, quizás, con el devenir del tiempo, otros autores lo han transcrito como Ana María, al ser más habitual. 


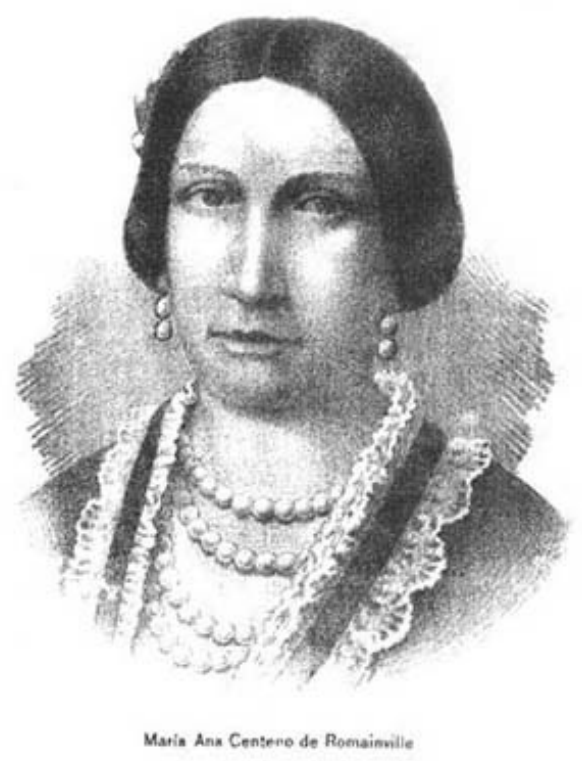

Fig. 1. Retrato de María Ana Centeno de Romainville. Fotografia: Matto de Turner, Clorinda (1890). Bocetos a lápiz de americanos célebres. Lima: Imprenta Bacigalupe, p. 194.

María Ana regresó a Cuzco en 1841, y en 1842 contrajo matrimonio con Pedro Romainville, uno de los primeros viajeros franceses que llegaron a Cuzco en la década de 1830 (Vilela Galván y De la Puente, 2001, p. 158). Al parecer, este enlace no fue bien avenido por su padre, ni tampoco por la sociedad cuzqueña. María Ana pertenecía a una familia distinguida, junto a sus hermanos Juan y Josefa, y eran muchos los peruanos que ambicionaban casarse con ella, con lo que su unión fue vista como "el descenso de estatus de quien había sido cortejada por 'hijos del Perú de distinguida posición"' (Matto de Turner, 1890, p. 200). El parisino Pedro Romainville recién acababa de llegar a Perú, en un tiempo en que los "forasteros, 'seres distintos y desnudos de religión', eran vistos no sin sospecha” (Rénique, 2015), al tiempo que era por entonces solo un pequeño comerciante (Vilela Galván y De la Puente, 2001, p. 159).

Esta unión, no obstante, pudo influir positivamente en la definición de una de las grandes pasiones de María Ana: el coleccionismo; pues probablemente Pedro Romainville tendría ciertas ideas de carácter ilustrado, según las cuales, el interés por los objetos del pasado, las curiosidades, o los generalmente denominados artificialia debía fomentarse. Del mismo modo que las relaciones con la familia que quedó en Europa podrían ayudarle a conseguir muchas de las piezas (Gänger, 2014a, p. 117), e incluso es de destacar cómo el esposo compartía su afición por el coleccionismo, y enseñaba sus piezas a los extranjeros que pasaban por allí, como su compatriota el conde Francis de Castelnau (Gänger, 2014a, p. 123). 
En 1847, María Ana quedó viuda con dos hijos muy pequeños, Eduardo y Adolfo Romainville Centeno -nacidos en 1845 y 1847-, por lo que desde ese momento ella sola debió hacerse cargo de su educación y manutención (García y García, 1925, pp. 253-254). Elvira García y García deja entrever que su situación económica distaba mucho de ser fácil tras la muerte de su esposo; si bien, no llegamos a concebir totalmente esa realidad, teniendo en cuenta que María Ana procedía de una familia bien avenida, ella misma actuaba como una dama de alta alcurnia y sus hijos heredaron un importante número de terrenos. Por ejemplo, la Hacienda Huadquiña, "más de seis veces el área del París que Pedro Romainville había dejado atrás para siempre" (Vilela Galván y De la Puente, 2001, p. 159), y que con el paso de los años terminará en manos de Tomás Alvistur, conocido coleccionista que se vio involucrado con personajes de la talla de Hiram Bingham (Vilela Galván y De la Puente, 2001, p. 159).

Uno de los hechos más destacados con respecto a la posible riqueza de la familia Romainville-Centeno, sería la compra de la finca Pucuto, en Andahuaylillas, provincia de Quispicanchis. Según aporta Luis Miguel Glave, María Ana compró la finca en 1853, pagando por ella 30.000 pesos (Glave, 1986, pp. 151-152); mientras que Clorinda Matto de Turner establece su compra un año después, en 1854, uniendo la herencia dejada por su marido a unos pequeños ahorros que ella misma había reunido (Matto de Turner, 1890, p. 202). Fechas aparte, lo importante es que su finca Pucuto se convirtió en albergue para los extranjeros que llegaban al Cuzco: "era una especie de hotel gratuito, para todo el que quisiera ocuparlo, no importaba que fuese desconocido y para esa alma caritativa no existía ni lejanamente la idea de la recompensa" (García y García, 1925, p. 254).

Esta circunstancia resultará crucial para el desarrollo y el futuro de su colección, ampliando su círculo de amistades más allá de su posición o estatus original (MacGregor, 2007, p. 66), y motivando que en su casa pernocten personajes muy conocidos como Ephraim George Squier, Paul Marcoy, y el mencionado conde de Castelnau (Mould de Pease, 2003, p. 62). Como suele ser habitual en este tipo de casos, (MacGregor, 2007, p. 69), la casa de María Ana Centeno se convirtió en lugar de reunión social, con vecinos de la élite de la ciudad de Cuzco, u otras personalidades procedentes de distintos países: "supo crearse el mejor salón que tuvo la sociedad cuzqueña, siendo su casa el centro de la ilustración” (Matto de Turner, 1890, p. 196). Su Museo sirvió como salón para veladas literarias (Guevara Gil, 1997, p. 184), y por sus dotes sociales, Clorinda Matto la comparó con Madame Geoffrin, organizadora de un famoso salón literario en el París del siglo XVIII (Matto de Turner, 1890, p. 196); otorgándole así importancia a su actividad, digna de relacionar con personajes de la élite europea, al mismo tiempo que su labor queda enmarcada en un espacio netamente femenino, tal como correspondía a una mujer con su educación y estatus.

La personalidad de María Ana Centeno se define tanto por sus dotes sociales como por su interés de ayudar al prójimo. Es considerada como una "dama de caridad", ejerciendo un maternalismo social desde su espacio doméstico (Peluffo, 2004, 
p. 111). Clorinda Matto la compara con san Vicente de Paul, o con Mary Carpenter, fundadora de una escuela para niñas y niños con pocos recursos, y ferviente defensora de la abolición de la esclavitud (Matto de Turner, 1890, p. 196). La misma autora ensalza a María Ana a la categoría de mujer ejemplar, modelo de virtud republicana, en el ejercicio de la caridad (Peluffo, 2004, p. 107). La "señora de Pucuto" -tal como Clorinda Matto se refiere a ella- es caracterizada en contraposición a los "arrebatos nativos"; en una esfera distinta a la de los indígenas y dentro de un universo femenino, donde las cualidades virtuosas debían sobresalir, incluso por encima de otras actividades como la coleccionista.

Así, sirvió de gran ayuda durante la epidemia de peste que asoló Cuzco en 1855, e igualmente, dedicó parte de sus esfuerzos a la defensa de la "raza indígena" (García y García, 1925, p. 254) en un encuentro con la otredad racial (Peluffo, 2004, p. 108). Nuestra protagonista es "expresión de la contención y la virtud imprescindibles para canalizar hacia el orden las grandes energías - no solo sociales sino también eróticasque bullen en el mundo indígena" (Rénique, 2015). En una labor sobre la que no nos resistimos a mencionar nuevamente las palabras de Clorinda Matto, quien al mismo tiempo que alaba sus acciones y la caracteriza como una persona muy cultivada, sitúa a María Ana en inferioridad con el universo masculino de su misma época:

“iiiCuántas veces la escuché, aún niña, consolar al afligido con palabras llenas de la santa unción caritativa, y la vi derramar lágrimas a la contemplación del infortunio ajeno!!! Ella, que poseía una esmerada educación nutrida por la frecuente lectura que la aleccionaba, en las ciencias; ella que fue mujer, no podía dejar de poseer esa exquisita sensibilidad que tan alto habla en favor del sexo débil" (Matto de Turner, 1890, p. 197).

María Ana Centeno comenzó a formar su colección en 1832 (Guevara Gil, 1997, p. 175), en una "de aquellas pasiones caprichosas rayanas en la locura" (García y García, 1925, p. 255), que denotan su posición social privilegiada, y su deseo de legitimar su rango, al poseer el dinero y los contactos suficientes para adquirir piezas de distintas partes del mundo (Gänger, 2014a, p. 117). Ella misma, en una visita realizada por Ricardo Dávalos y Lissón en la década de 1870, afirmó que su intención había sido la de concebir una colección de "maravillas", mediante la recopilación de elementos peculiares, pues no había curiosidad que no quisiera poseer en su casa (Gänger, 2014a, p. 115). De tal manera que generó un gabinete de curiosidades o cuarto de maravillas, al estilo de aquellos formados en Europa siglos atrás, los cuales conformaban en sí mismos un "microcosmos" del mundo conocido, con objetos que despertaban la fascinación y la curiosidad, piezas de civilizaciones antiguas, o los llamados artificialia (Yaya, 2008, p. 173-174), recopilados por impulso y azar más que por la existencia de un diseño premeditado (MacGregor, 2007, p. 11).

Esta colección -marcada por la curiosidad, el exotismo y la búsqueda de lo maravilloso-, y en la que encontramos una importante presencia del componente precolombino, fue una de las grandes pasiones de María Ana, ocupando varios salones en 
su casa familiar (Armas Asín, 2019). Incluso en sus últimas disposiciones al acercarse su muerte, ordenó que durante el velatorio su cuerpo fuese colocado en el salón junto a sus antigüedades (García y García, 1925, p. 256). María Ana fallece en 1874 rodeada por una colección que ya no podemos disfrutar de manera conjunta pero que, por su importancia y representatividad, nos gustaría que se conociese mucho mejor, también como un modo de otorgar a la señora Centeno el lugar que le pertenece en la historia y la historiografía.

\section{EL GABINETE DE GURIOSIDADES Y ANTIGÜEDADES PRECOLOMBINAS}

María Ana Centeno lleva a cabo su colección en una coyuntura inmersa en su tiempo y sus circunstancias, pues si bien comienza a considerar las obras precolombinas como algo más que testimonios relacionados con culturas desaparecidas, aún se deja llevar por la curiosidad y la concepción de lo maravilloso. En su gabinete era posible encontrar obras de distintas épocas, culturas y lugares, sin seguir aparentemente un criterio científico para su ordenamiento y clasificación; probablemente, al igual que los gabinetes de siglos anteriores, los objetos estaban dispuestos según su naturaleza, siguiendo un criterio estético, o con el objetivo de generar un impacto visual en el espectador (Yaya, 2008, pp. 181-182). De hecho, uno de los visitantes de la colección comentó que las piezas se encontraban todas repartidas por la habitación, unas encimas de otras, resultando complicado poderlas disfrutar de manera individual (Gänger, 2014a, p. 110). Según escribió el periodista limeño Dávalos y Lissón en 1875, Centeno no había comenzado a clasificar su colección porque murió antes de considerar que esta estuviese culminada (Gänger, 2014a, pp. 112-113). Con lo cual, aunque María Ana conocía muchos detalles sobre sus piezas, nunca llegó a ponerlos por escrito, tal como hicieron algunos coetáneos como Emilio Montes o José Lucas Caparó (Gänger, 2014b, pp. 56-57).

Ese cometido recayó en sus hijos, que tan solo dos años después de la muerte de María Ana, encargaron la realización de un catálogo (Gänger, 2014a, p. 110), marcando así la identidad y significación de la colección (Vázquez, 2001, p. 24), el cual sintetiza en sí mismo varias tendencias de la época. El catálogo presenta a las piezas desde el prisma de la otredad, calificándolas como curiosidades o maravillas; y su realización probablemente responde a una querencia generalizada en el Cuzco de la época, donde además de vender algunas piezas sueltas, era especialmente destacable la compra-venta de colecciones ordenadas, descritas y con sus correspondientes catálogos (Gänger, 2014b, p. 91)

Del Catálogo del Museo de la señora Centeno (Lima: Imprenta de la Merced, 1876) nos interesa sobre todo la enumeración de obras calificadas como "antigüedades", más de mil, mayoritariamente procedentes de las culturas precolombinas del país: platos, vasijas, figuras de madera, piezas de piedra, metales preciosos, adornos, $\mathrm{o}$ 
una momia vestida a la usanza de su época. Incluso tenía joyas que decían pertenecer a los nobles del Imperio Inca, entre ellas un mascapaycha de oro (Gänger, 2014b, p. 28) -una de las más importantes insignias de los incas, y que a lo largo de los años recibió tratamientos diversos, hasta convertirse en el caso de la colección que tratamos en un objeto relacionado con la arqueología y la antigüedad (Gänger, 2014b, p. 44)-. Eran piezas pertenecientes principalmente a la cultura inca, $u$ otras desarrolladas en la zona de Cuzco, aunque también era posible encontrar obras procedentes de la costa norte peruana (Gänger, 2014a, p. 113). Lo cual nos demuestra cómo el tráfico y el movimiento de piezas en el seno del mismo país es una realidad desde fechas tempranas.

La colección estaba dotada de minerales, piedras procedentes de distintos lugares, e incluso parte de un mosaico romano; junto a objetos pertenecientes a la época de conquista española, pinturas religiosas españolas y francesas, o monedas. También porcelanas japonesas y chinas, metales de China, además de telas y ropas de estos lugares exóticos. Todos ellos símbolos de un mundo quizás mucho más globalizado de lo que a priori podríamos imaginar, que permitía la llegada a un lugar como Cuzco -no precisamente destacable por su accesibilidad en aquellos años- de mercancías de todo tipo (Gänger, 2014a, p. 117).

El componente natural era igualmente destacable, con casi un centenar de pájaros disecados, mariposas, insectos, reptiles, serpientes o peces; e incluso tenía rarezas, como una gallina con tres patas, fetos de oveja, o el cabello de un albino, pues en este tipo de colecciones lo insólito y fantástico ejerce cierta fascinación, ya presente en algunos gabinetes del siglo XVI (López Rodríguez, 1995, p. 76), y que los relacionaba directamente con un mundo de leyendas y ensueños (Yaya, 2008, p. 174). Además, María Ana se sentía atraída por los objetos realizados por las sociedades indígenas actuales, algunos de los cuales fueron catalogados como pertenecientes a los "salvajes” de Paucartambo, Santa Ana, Marcapata y Lares (Gänger, 2014a, pp. 110-111). Ello como curiosidad rayana en el exotismo, pues a los habitantes del Amazonas se les veía efectivamente como a salvajes, que vivían de manera muy distinta a la clase alta cuzqueña a la que Centeno pertenecía (Gänger, 2014a, p. 114).

Sobre la recepción de su colección en época más o menos coetánea, encontramos varias referencias que la describen como variada y caracterizada por la curiosidad. Clorinda Matto de Turner dedicó unas elogiosas palabras a la colección; y otra descripción, de 1925, la encontramos en la obra de Elvira García y García, quien la calificó como un verdadero museo arqueológico, en el que era posible seguir parte de la historia del país y nos narra cómo María Ana tenía en su poder:

"Notables mosaicos de los principales palacios italianos; trozos de lava; petrificaciones de toda clase; porcelanas de los Sevres; tapices y encajes de las más ricas fábricas; retratos espléndidos de casi todos los Presidentes del Perú, de su familia y de las personas ilustres del Cuzco; cuadros al óleo de firmas renombradas; figuras de pasta trabajadas 
en el país; colecciones riquísimas hechas en piedra; vasos, vasijas, instrumentos, cátaros, morteros e infinidad de cosas hechas en piedra, acusando las diferentes civilizaciones antiguas; trabajos hechos en barro, imitando todo lo que los hombres, de las diferentes zonas han hecho en oro, bronce, hierro, mármol, etc., etc.; utensilios de familia; adornos caprichosos y menaje de los Incas; tejidos, dibujos, geroglíícos, trabajos de oro y plata, alhajas, cetros" (García y García, 1925, p. 255).

Podemos así entrever cómo, tan solo unos años tras la muerte de nuestra protagonista, e incluso durante su vida, su colección era elogiada desde distintos círculos. Se puede igualmente intuir cierto nacionalismo en las palabras de sus compatriotas (Gänger, 2014a, p. 113), como parte de una tendencia más amplia hacia la construcción de una identidad de la nueva nación, y la necesidad de reafirmar las actitudes propias. Desde círculos de intelectuales y artistas se otorgaba especial importancia a las piezas de las culturas precolombinas -especialmente a las incaicas en el caso de Cuzco- como una reivindicación del Tawantinsuyo y el pasado del país (Kuon Arce et al., 2009, p. 25).

No debemos olvidar que María Ana Centeno formó su colección en las décadas centrales del siglo XIX, en un momento históricamente convulso, y marcado por acontecimientos de gran importancia, entre los que debemos resaltar la consecución de la Independencia en 1821. La aparición de Perú como país independiente marcó el modo en que se consideraba a las antigüedades pertenecientes a los pueblos precolombinos, pues la creación de una nueva nación llevó a la búsqueda de símbolos identitarios. Con lo cual, las ruinas, monumentos y piezas pertenecientes a estos pueblos comenzaron a convertirse en un elemento esencial, como símbolo de un "pasado glorioso". De alguna forma, lo precolombino se vincula con una visión "moderna" de distintos países americanos (Gutiérrez Viñuales, 2013, p. 89), entre ellos Perú.

Este no fue un proceso inmediato, sino que gradualmente se fueron definiendo los principios identitarios de la nación; y se produjo un mayor interés por el pasado prehispánico, por su estudio, protección y, en lo que más nos atañe, por su coleccionismo. Este último era tanto de carácter privado, como institucional a partir de la creación de los museos nacionales, centros necesarios para definir la identidad de la nueva e incipiente república. En Perú, en los primeros años tras la Independencia, se resolvió la creación del primer Museo Nacional (Ravines, 1996, p. 157); no obstante, la aparición temprana de esta institución no debe relacionarse con una inmediata concepción patrimonial, que todavía debía continuar madurando; e incluso era bastante común que las colecciones particulares fuesen mucho más prolijas que las públicas, y en lo que concierne a la de María Ana, José Mariano Macedo mencionó cómo su adquisición sería un importante acicate para el Museo Nacional (Riviale, 2015a, p. 91).

En la época que tratamos todavía se conoce muy poco sobre las antigüedades precolombinas, que apenas comienzan a ser estudiadas y consideradas con un crite- 
rio científico. En 1847 la publicación de la obra History of the Conquest of Peru, de William H. Prescott, llevó a investigadores extranjeros a explorar el país para reunir colecciones de objetos prehispánicos (Ravines, 1989, p. 18). Y en 1851 se publica Antigüedades Peruanas, de Mariano Eduardo de Rivero y Johann Jakob von Tschudi, la primera síntesis de la arqueología del país (Ravines, 1996, p. 29). En 1860, la arqueología peruana "alcanzó su vigencia" (Ravines, 1989, p. 18) con personajes como Ephraim George Squier, quien en su obra Peru. Incidents of travel and explorations in the land of the Incas, además de mencionar la visita realizada a María Ana Centeno, realiza un inventario de monumentos arqueológicos, suponiendo un importante acicate para el coleccionismo de obras andinas, sobre todo a partir de su publicación en Inglaterra y Estados Unidos (Bruhns y Kelker, 2010, p. 92). A Squier le siguen otros muchos viajeros y exploradores, como Wilhem Reiss y Alphons Stübel, quienes viajaron a Perú bajo el patrocinio del Museo Etnológico de Berlín para recoger materiales y realizar excavaciones (Tantaleán, 2016, p. 42). De esta forma, el estudio de las antigüedades prehispánicas estaba aún en una etapa de tránsito entre "la contemplación artística o exótica de los monumentos y las artesanías, hacia su integración como testimonios de la historia" (Lumbreras, 1998, p. 178); y atraía a los extranjeros anteriormente mencionados, y poco a poco también a los propios peruanos.

En el Cuzco del siglo XIX, además de la colección de María Ana, encontramos el acervo de la familia Bennet, realizado a instancias de Astete de Bennet, que se consideraba a sí misma como heredera de "las guerreras de Pizarro" (Gänger, 2014b, p. 49). También el Museo Público y Biblioteca, fundado en 1848 por José Miguel Medina (Armas Asín, 2019) y que recibió donaciones de eminentes ciudadanos y ciudadanas (Gänger, 2014b, p. 49). Otro caso sería el Museo de Antigüedades Peruanas e Inkaikas, fruto de la iniciativa de Emilio Montes de Segura y Aldazabal y que en 1892 tenía casi dos mil objetos, con momias, y piezas de piedra, metales, cerámicas o tejidos (Ravines, 1996, p. 183), muy en relación con el llevado a cabo por nuestra coleccionista, pues el Imperio Inca estuvo evidentemente muy presente en ambos acervos, como parte de un pasado venerable y glorioso. De hecho, la colección atesorada por Emilio Montes guardaba diversas similitudes con la de María Ana, ejemplificadas en la presencia de momias, keros, cerámicas como aríbalos, o fósiles (Gänger, 2014b, p. 21-24). De su colección, Montes realizó un catálogo en el que demuestra su amplio conocimiento sobre las culturas precolombinas, e incluso compara sus objetos con aquellos procedentes de Grecia y Roma, realizando un primer reconocimiento estético de los elementos precolombinos, acercándolos en plano de igualdad a las consideradas antigüedades por excelencia (Gänger, 2014b, pp. 59-63).

De fechas un poco más avanzadas, no debemos dejar de destacar el Museo de Antigüedades Peruanas de José Lucas Caparó Muñiz, que en 1919 contaba con más de dos mil piezas, cuya calidad y representatividad le llevó a recibir distintas ofertas de compra, para terminar en la Universidad San Antonio Abad del Cuzco (Guevara Gil, 1997, pp. 177-181). Esta es una de las colecciones más relevantes, cuyo autor llegó a comparar con "el recordado [Museo] de la Sra. Centeno con 10 secciones 
menos" (tomado de: Guevara Gil, 1997, p. 171), evidentemente enalteciendo la suya propia. Las similitudes entre ambas colecciones resultan evidentes, con la presencia de keros, piezas de cerámica prehispánica, además de porcelanas chinas y japonesas (Gänger, 2014b, pp. 18-20), y otros muchos objetos procedentes de distintas geografías, entre las que destacaba la presencia incaica. No en vano, tanto Caparó como Centeno formaban parte de una élite que se veía a sí misma como los "guardianes" e intérpretes del mundo indígena (Gänger, 2014b, p. 35), en una visión paternalista donde el propio acto de coleccionar les aseguraba una primacía social y cultural más allá de su contexto local.

La formación de colecciones se relaciona con una tendencia globalizada, que reafirmaba a su poseedor como parte de un universo ilustrado, formado por personajes de alta alcurnia que buscaban legitimar su posición social (Gänger, 2014b, p. 25, MacGregor, 2007, p. 21), así como sus identidades nacionales y regionales (Vázquez, 2001, p. 10). De este modo, ya en el siglo XVI era posible encontrar en ciudades como Sevilla colecciones con objetos procedentes de América, como por ejemplo la de Nicolás Monardes, o la atesorada por Rodrigo Zamorano (López Rodríguez, 1995, pp. 78-70, 86-87). No obstante, debemos tener en cuenta que el siglo XIX y, sobre todo, el $\mathrm{XX}$, conllevó el "despertar" de una conciencia mucho mayor sobre la importancia de las obras artísticas, tanto en Perú, como en distintas partes del mundo. Un ejemplo de ello lo encontramos en Madrid, donde surge la Sociedad de Amigos del Arte, formada por ricos poseedores de colecciones, pertenecientes a una burguesía acomodada que tenía acceso a las obras artísticas (Gkozgkou, 2013, pp. 103-105); una institución paralela a la formada en Cuzco en 1868, la Asociación Arqueológica Peruana, igualmente con personajes de la élite que se reunían para compartir sus impresiones, principalmente sobre las obras prehispánicas que recién estaban comenzando a encontrarse (Gänger, 2014b, p. 30).

Con todo ello, las colecciones cuzqueñas son símbolo de una cultura cosmopolita formada por eruditos y personajes de alta alcurnia que leían los mismos libros y hablaban entre ellos de sus adquisiciones (Gänger, 2014b, pp. 59, 67); por lo que prácticamente todos los miembros de la élite local poseía antigüedades precolombinas, principalmente incaicas (Gänger, 2014a, p. 116). Las piezas prehispánicas se consideraban dignas de conservar y exponer en sus hogares, tanto por suscitar la curiosidad de los viajeros extranjeros que comenzaban a pasear por las calles de Cuzco, como por representar a un indigenismo (o incaísmo), cada vez más latente (Armas Asín, 2019). Según afirmaba José Tamayo Herrero:

"Los "mistis" [mestizos] surandinos y sobre todo la intelligentsia regional, despertaron a través de los museos la conciencia de una herencia gloriosa que permanecía ignorada; empezaron a sentir el incario como su propio y grandioso pasado. Por eso estos museos del siglo XIX, reveladores de un gusto estético nuevo y nacional, cumplieron un papel pedagógico, no por silencioso, menos importante" (tomado de: Kuon Arce et al., 2009, p. 50). 


\section{VENTA Y DISPERSIÓN DE LA COLEGGIÓN: ¿A QUIÉN PERTENECE EL PATRIMONIO?}

La colección realizada por María Ana Centeno cumplía todos los requisitos para ser un codiciado objeto de deseo. Tanto para los ciudadanos de Cuzco, como para los extranjeros, que la encontraban ante todo destacable por el componente "indígena" y la presencia de antigüedades precolombinas. Uno de estos personajes será Ephraim George Squier, quien se alojó en casa de nuestra protagonista, y tuvo el privilegio de conocer la colección, diciendo de su anfitriona que era "la coleccionista del más fino y más valioso museo de antigüedades del Perú" (tomado de: Armas Asín, 2019). Él mismo cuenta cómo María Ana estaba acostumbrada a recibir visitas y compartir con sus huéspedes las historias sobre las piezas de su colección (Gänger, 2014a, p. 120 ), en una tendencia aperturista que también observamos en otros lugares de Europa, y que servía como legitimación social para su poseedora (Vázquez, 2001, pp. 7-8). E incluso, entre sus objetos había un cráneo con el hueso frontal trepanado que regaló a Squier para que lo investigara, considerando que "en los Estados Unidos la ciencia estaba más avanzada que aquí" (tomado de: Mould de Pease, 2003, p. 62).

Esta última es una afirmación que enlaza con el propio sentir de la época por dos razones principales: en primer lugar, aunque se trata de restos humanos, el cráneo trepanado resulta una curiosidad, digna de coleccionar y estudiar; y, en segundo lugar, estamos tratando una época en la que se consideraba que naciones como Perú no estaban lo suficientemente avanzadas como para realizar estudios arqueológicos por cuenta propia. De este modo, los viajeros y arqueólogos que andaban por tierras peruanas consideraron correcto y "una buena opción" el transporte de obras a sus lugares de origen, donde se conservarían, estudiarían y valorizarían mucho mejor.

No es de extrañar que en 1880 (Mould de Pease, 2003, p. 62), 1887 (Gänger, 2014a), o 1888 (Armas Asín, 2019; Guevara Gil, 1997, p. 175; Fischer, 2015), según la fuente consultada, buena parte de la colección fuese vendida por sus descendientes al Museo Etnológico de Berlín (en alemán: Ethnologisches Museum), tras la muerte de María Ana y tras exponerse durante un par de años en las mismas circunstancias en las que ella misma la dejó. Principalmente, desde Berlín mostraron interés por las obras prehispánicas, mientras que otras muchas piezas de la colección permanecieron en manos de sus herederos, y hoy día poco se conoce sobre su paradero (Gänger, 2014a, p. 119).

El artífice de la venta fue Adolf Bastian, primer director del Museo Etnológico de Berlín, quien tuvo ocasión de conocer la colección unos años antes de la muerte de María Ana y, aunque ella se resistía a venderla (Fischer, 2015, p. 28), calificó su obtención como una "cuestión vital" para el Museo berlinés (Fischer, 2015, p. 30). Para su adquisición finalmente hubo de esperar algunos años, y gracias a la ayuda de la embajada alemana en Perú y tras competir con otras instituciones interesadas, pudo adquirir 1000 piezas (de las aproximadamente 1500 que tendría la colección) por el valor de 48.000 marcos (Fischer, 2015, p. 30). Entre ellas, el mencionado mascapay- 
cha de oro, del que sin embargo no se realiza referencia directa en el catálogo (quizás podría ser un "adorno de oro"), y del que tampoco tenemos noticias en la actualidad: ha podido ser fundido, ser víctima de un robo o venta ilegal, o incluso es posible que siga en la propia institución sin ser identificado (Gänger, 2014b, p. 98). Con lo cual, el conjunto al completo no ha llegado hasta nuestros días y, evidentemente, sus piezas han adquirido un significado nuevo.

Al visitar el catálogo en línea del Museo Etnológico de Berlín (Catálogo, 2020), podemos encontrar que quedan aproximadamente quinientas piezas precolombinas adscritas a la colección Centeno. Entre ellas, hallamos algunas de la cultura moche -como una figura antropomorfa sentada (Fig. 2)-, o de las culturas chimú o Ica. Evidentemente, el grueso de la colección está compuesta por obras pertenecientes a la cultura inca, como aríbalos (Fig. 3), conopas, o keros (Figs. 4 y 5), piezas de piedra, cerámicas (platos, cuencos...), o incluso un uncu de periodo colonial, datado en el siglo XVI. También nos llama la atención la presencia de un par de figuras de oro - quizás demasiado pocas, teniendo en cuenta que en época de María Ana todavía se asimila la tasación de una pieza a su valor en oro o plata-, además de un tupu de plata, y una máscara realizada con piel de llama. Del mismo modo que la colección contempla lo que podríamos considerar como artesanías, con collares y pulseras realizadas con semillas y restos de frutos.
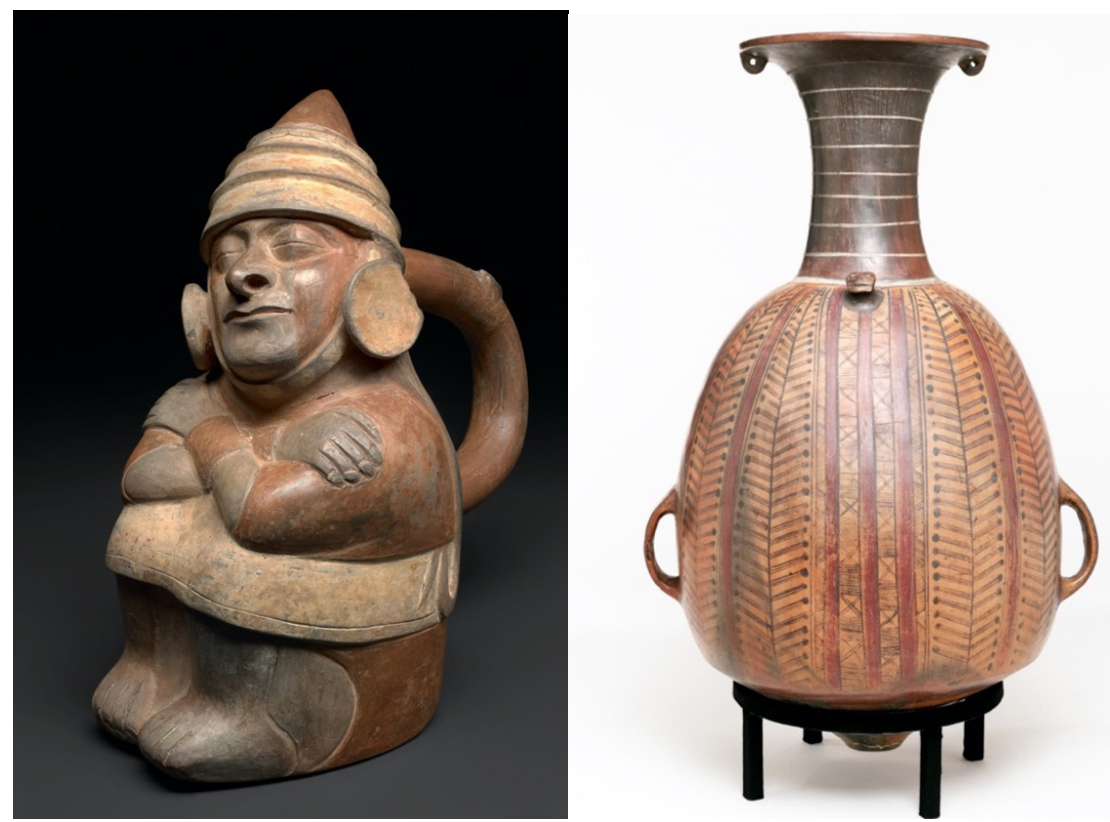

Fig. 2 (izq.). Figura sedente antropomorfa. Cultura moche. Colección María Ana Centeno. Staatliche Museen zu Berlin, Ethnologisches Museum, V A 8033, fotógrafo: Clau: dia Obrocki. Fig. 3 (dcha.). Aríbalo. Cultura inca. Colección María Ana Centeno. Staate liche Museen zu Berlin, Ethnologisches Museum, V A 7890, fotógrafo: Martin Franken. 


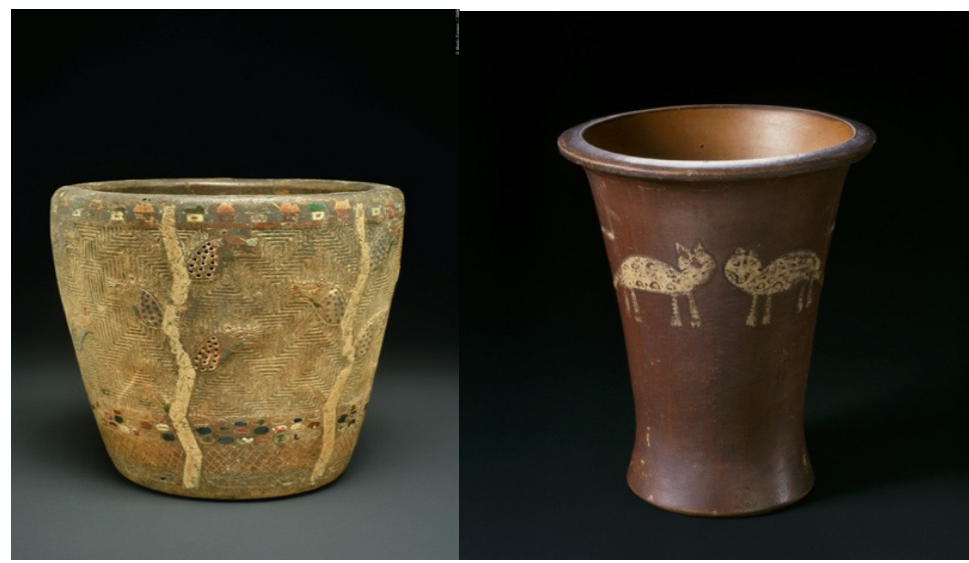

Fig. 4 (izq.). Kero. Cultura inca. Colección María Ana Centeno. Staatliche Museen zu Berlin, Ethnologisches Museum, V A 8110, fotógrafo: Sandra Steiß. Fig. 5 (dcha.). Kero. Cultura inca. Colección María Ana Centeno. Staatliche Museen zu Berlin, Ethnologisches Museum, V A 8915, fotógrafo: Martin Franken.

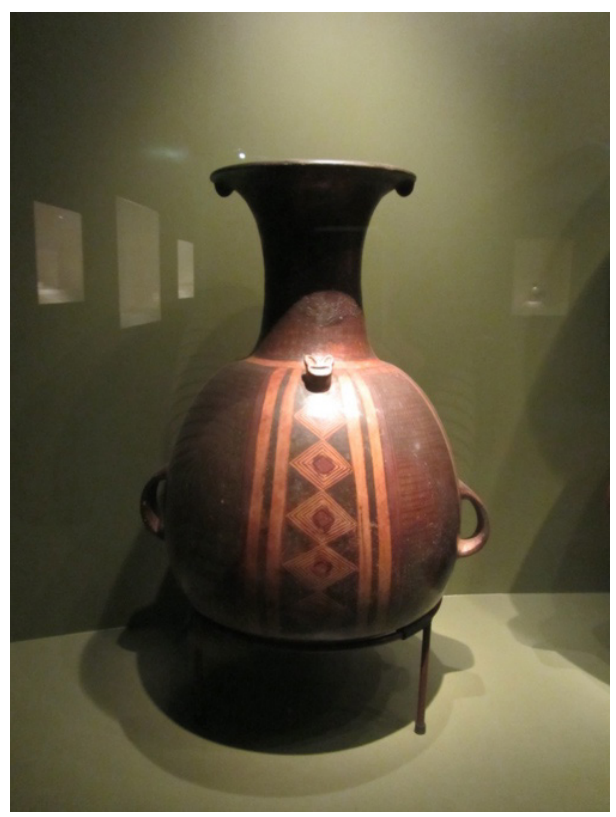

Fig. 6. Aríbalo. Cultura inca. Museo de Arte Precolombino (Cuzco, Perú). Fotografía de la autora, 2017.

Se trata de un conjunto de piezas representativas de su tiempo y sus culturas, que podemos encontrar en otros museos de Perú y el resto del mundo. Por ejemplo, aríbalos incaicos muy parecidos poseen instituciones cuzqueñas actuales como el 
Museo de Arte Precolombino (Fig. 6), o el Museo Machu Picchu de la Casa Concha. Lo mismo podríamos decir de las conopas, piezas normalmente con forma de llama o alpaca que encontramos en un importante número de instituciones, entre ellas, el Museo de Arte Precolombino (Fig. 7). Sería también el caso de una de las figuras de oro de la colección Centeno (con número de inventario V A 8653), de la que hallamos piezas semejantes en el Museo Machu Picchu de la Casa Concha (Fig. 8), o en el Museo del Banco Central de Reserva del Perú en Lima.

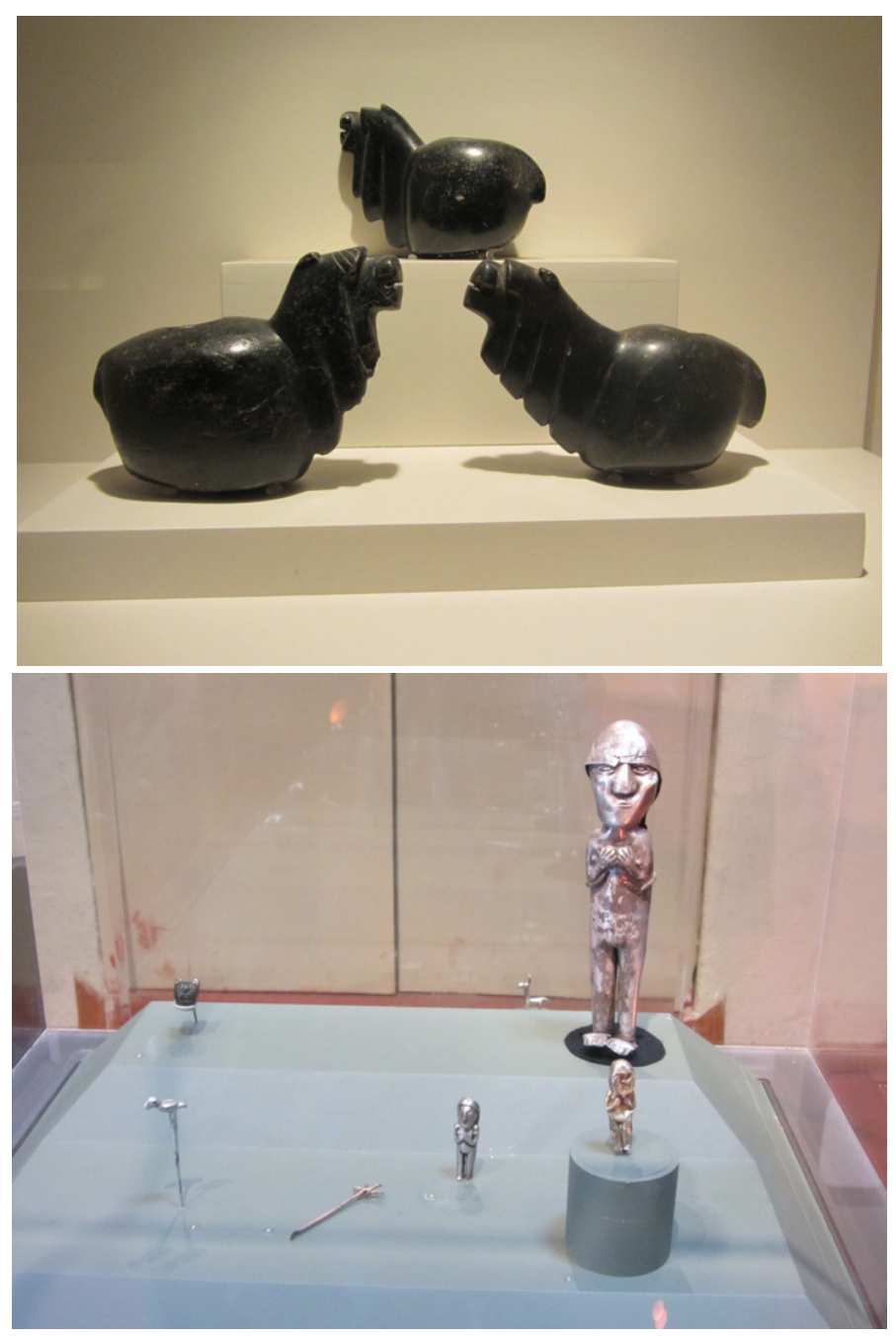

Fig. 7 (arriba). Conopas. Cultura inca. Museo de Arte Precolombino (Cuzco, Perú). Fotografia de la autora, 2017. Fig. 8 (abajo). Piezas metálicas con forma antropomorfa. Museo Machu Picchu de la Casa Concha (Cuzco, Perú). Fotografía de la autora, 2017. 
Con lo cual, teniendo en cuenta su contenido, habría que considerar de manera individual el posible regreso de estas obras a su lugar de origen, una vez que la colección Centeno ha sido dividida y sería muy complicado (o incluso prácticamente imposible) reunirla de nuevo. Es decir, debemos tener en cuenta el hecho de que muchas de las piezas presentes en Berlín no son realmente únicas, sino que de ellas ya se posee una muestra representativa en su lugar de procedencia, por lo que quizás resulta una buena opción su permanencia en otras instituciones, dado que la dispersión controlada del patrimonio sí resulta deseable.

Estamos de acuerdo con el internacionalismo cultural (Merryman, 1986) al afirmar que el patrimonio pertenece a la humanidad en su conjunto pues, al fin y al cabo, las piezas son embajadoras y pueden resguardarse en distintos lugares. E incluso podría considerarse que la colección también forma parte de la historia de Alemania y, concretamente, del Museo Etnológico de Berlín, lugar que la ha custodiado desde finales del siglo XIX. Eso sí, debemos reconocer a los países defensores del nacionalismo cultural (Merryman, 1986) una serie de condiciones y no permitir que la presencia de una colección en una institución extranjera suponga una "ofensa" para su lugar de origen. Al mismo tiempo que se debe prestar especial atención al hecho de que nunca sean piezas adquiridas recientemente, en una medida que intentaría frenar el actual saqueo de bienes culturales.

La presencia universalista o internacionalista de piezas precolombinas en distintos puntos del mundo debe regirse por un estricto código moral -aunque por su propia naturaleza sea complicado de establecer-. Según el cual, las piezas realmente significativas regresen a su lugar de origen, pues su ausencia genera un importante hándicap en el lugar del que proceden, y servirían para representar un momento o acontecimiento de la historia del país. Ante lo que nos preguntamos: ¿sería este el caso del (desaparecido) mascapaycha, o alguna otra pieza de la colección?

Quizás lo ideal en este tipo de circunstancias -además de la devolución de las piezas más representativas- sería que la institución depositaria de los objetos realizara una reparación de tipo moral o intelectual, reconociendo de dónde proceden los objetos y en qué circunstancias se recogieron. Pues la compra de esta colección se realizó en un contexto marcado por el colonialismo, en el que la ventaja del europeo quedaba bastante latente, y donde podemos observar una clara falta de consciencia en torno a la valía de la colección, por parte de los herederos de María Ana, artífices de la venta. Esta última es una tendencia bastante usual, y que podría extrapolarse a otras realidades como la española, que durante todo el siglo XIX y parte del siglo XX se caracterizó por "una conciencia pública y privada bastante laxa en lo referente a los bienes patrimoniales fuera de España", e incluso era común que la venta en alza de una pieza en el extranjero generase satisfacción entre los compatriotas, aumentándose así el propio prestigio nacional (Gkozgkou, 2013, p. 114).

En la época que tratamos, solo unos pocos intelectuales fueron conscientes de lo que la migración patrimonial suponía para sus naciones. Así, la venta de esta co- 
lección significó una pérdida patrimonial que José Tamayo Herrera calificó -junto a la de la colección del Museo Montes- como una "descapitalización cultural de la región” (tomado de: Guevara Gil, 1997, p. 175). Y ya Elvira García y García expuso en 1925:

"No pobres los herederos de la señora Centeno; pero sin conciencia suficiente, para apreciar cuánto valía y lo que significaba para el propio país esa hermosa colección, sin rival en todo el Perú, ha pasado a manos extranjeras, que se han llevado en pequeñas fracciones, lotes valiosos, que han sido entregados, sin saber lo que se hacía". (García y García, 1925. p. 256).

Tampoco debemos olvidar que la venta se llevó a cabo durante, o en plazos muy cercanos -según la que consideremos como fecha de la venta- a la Guerra del Pacífico (1879-1883) que enfrentó a Chile con Bolivia y Perú, un periodo especialmente virulento y negativo para el patrimonio peruano (Tantaleán, 2016, p. 43). Según expone Mariana Mould de Pease: la venta "se debió - muy probablemente- a urgencias económicas inmediatas y hoy se puede decir al respecto que es una temprana evidencia de cómo entre nosotros se fue perdiendo la visión de futuro y de intereses comunes nacionales" (Mould de Pease, 2003, pp. 62-63). Con lo cual, las propias circunstancias adversas vividas en la época en el país, junto a los pretendidos sentimientos colonialistas mencionados, provocaron la salida del conjunto reseñado hacia el extranjero.

Por supuesto, no serían estas las únicas piezas que marchan a otros lugares durante el siglo XIX, sobre todo, por el negocio tan lucrativo que suponía la venta de estas colecciones, que llevó a peruanos como el mencionado Emilio Montes, o los propios hijos de María Ana a vender la suya propia. Debemos situar estas transacciones en el seno de una tendencia coleccionista de carácter internacional que, en el caso de las antigüedades peruanas, respondía principalmente a dos razones: la fama particular del país, y el desafío científico que estas piezas implicaban (Riviale, 2015b). Con lo cual, con el paso de los años y a partir de su dispersión por distintos puntos del orbe, van obteniendo cada vez más valor.

En 1878 ya se exponen piezas precolombinas en el Museo del Trocadero de París, institución a la que Herman Anglada Camarasa organizaba visitas junto a otros compañeros precisamente para ver las colecciones americanas (Gutiérrez Viñuales, 2013, p. 91). En Madrid, en 1881 se celebra el IV Congreso Internacional de Americanistas, evento en el que se exponen vasos peruanos, momias y otros objetos procedentes de América Latina, en una exposición que sirvió para "avivar el interés por los objetos americanos" (Cabello Carro, 1989, p. 40). Algunos años más tarde, en 1933 y 1935 se expone en París y Madrid la colección peruana formada por Juan Larrea, con un catálogo que se anuncia como una exposición de "arte" inca. Estas obras se convierten igualmente en inspiración para artistas como Paul Gauguin, que de pequeño tuvo acceso a la colección formada por su madre, Aline Chazal (hija de Flora Tristán) (Majluf y Wuffarden, 1999, p. 103). Y en Perú, encontramos a José Sabogal, Francisco 
González Gamarra, Teófilo Castillo, o Elena Izcue, todos ellos vinculados con el indigenismo y la búsqueda de nuevos lenguajes a partir del pasado prehispánico.

Con lo cual, no es de extrañar que el componente precolombino se convirtiese en objeto de inspiración para artistas a ambos lados del océano, por tener acceso a las piezas, así como al trabajo de los propios artistas: europeos que viajan a América y viceversa (Gutiérrez Viñuales, 2013, p. 91). Este hecho tuvo una doble consecuencia o repercusión, pues al mismo tiempo que sustenta el reconocimiento como piezas artísticas de los restos del mundo precolombino, obras como las de Gauguin llegaban a un público inclusive mucho más numeroso, sentando parte de las bases de un arte inspirado en lo "primitivo" (Majluf y Wuffarden, 1999, p. 103), y generando un nuevo tipo de público deseoso de adquirir objetos prehispánicos para sus colecciones.

En definitiva, las piezas precolombinas ya desde el siglo XIX generan un negocio muy lucrativo, con un círculo de compradores cada vez más evidente, y un valor comercial más que notable -eran un "valor seguro ante los comerciantes de antigüedades" (Riviale, 2015b)-. Estas piezas podían comprarse o adquirirse mediante excavaciones ilegales, conocidas en Perú como huaqueo, en una actividad que se incrementa sobre todo a finales del siglo XIX y durante todo el siglo XX. Hasta el punto en que incluso en el mismo siglo XIX ya se realizaron las primeras falsificaciones, en previsión de la amplia demanda (Riviale, 2015b; Bruhns y Kelker, 2010), y en un momento en que el mercado de obras de arte en Europa comienza también a saturarse de piezas falsas (Vázquez, 2001, p. 49). Todo ello, con graves consecuencias para su país de origen, y que recoge Eugenio Larraburre y Unánue:

"Nosotros, los dueños de las inagotables fuentes que han producido esas reliquias históricas ¿qué poseemos? La respuesta es muy sencilla: ¡nada!

Desgraciadamente, las colecciones cuando llegan a formarse, duran poco tiempo entre nosotros y son motivo no de estudio sino de especulación.

Aquí, cansados estamos de verlo, llega cualquier viajero, toma una cuadrilla de peones y se echa a desenterrar momias y objetos, sin permiso de nadie, como si estuviese en casa propia, para no dejar más que el recuerdo de su paso" (Ravines, 1996, p. 163).

No debemos dejar de notar que ya existía una legislación al respecto, promulgada por el gobierno peruano desde la consecución de su Independencia. La primera ley de protección patrimonial se remonta al año 1822: Decreto Supremo n. ${ }^{\circ} 89$, de 2 de abril "Sobre la conservación de monumentos prehispánicos". El cual, en su preámbulo deja constancia de la necesidad de proteger los monumentos antiguos del país, sobre todo ante la circunstancia de que "con dolor se han visto hasta aquí vender objetos inapreciables, y llevarse a donde es conocido su valor, privándonos de la ventaja de poseer lo nuestro" (Ávalos de Matos y Ravines, 1974, p. 373). Y hacía hincapié en la prohibición de extraer objetos de las huacas sin licencia, y en la penalización de aquellos que incurrían en delito de huaqueo (Ávalos de Matos y Ravines, 1974, p. 373). 
En 1825, Simón Bolívar trató la protección de los monumentos incas en Cuzco, que debían ser considerados como "reliquias" de un pasado glorioso (Gänger, 2014b, p. 37); y en 1840 las autoridades locales intervinieron para regular y limitar las búsquedas de tesoros en la ciudad (Gänger, 2014b, p. 84). Con lo cual, para la fecha en la que María Ana Centeno entrega la pieza al E.G. Squier ya existía una incipiente legislación para proteger a los elementos pertenecientes al pasado precolombino. $\mathrm{Al}$ igual que para el momento en que se realiza la venta y exportación de la colección a Berlín, en la década de 1880.

Huelga decir que el decreto de 1822 no surtió de manera inmediata el efecto esperado, pues tal como se comentaba en la época, "a excepción de dos o tres personas, nadie ha solicitado el permiso indicado, y es público que continuamente se extraen dichos artículos para Europa" (Ávalos de Matos y Ravines, 1974, p. 376). Todavía hubo que esperar hasta contar con una legislación efectiva al respecto, que se fue ampliando con el paso de los años, y cuya ineficacia provocó que durante buena parte del siglo XX se pudiesen sacar obras, tanto de debajo de la tierra, como del propio país, de manera relativamente sencilla. Hasta el punto en que en 1947 aparece un decreto supremo en relación a la exportación de piezas arqueológicas, considerado "uno de los males que mayor y más constantemente amenazan a la conservación de estos tesoros" (Ávalos de Matos y Ravines, 1974, p. 434).

Según las evidencias anteriores, la venta de la colección Centeno se realizó en un momento en que ya existía una legislación patrimonial, cuya falta de aplicación y extensión entre la sociedad conllevaba serias dudas acerca de la pertenencia del patrimonio: “¿A sus propietarios? ¿ $\mathrm{O}$ a aquella abstracción, todavía en construcción, de la nación?” (Kagan, 2013, p. 194). Si bien, no podríamos realizar una reclamación formal de la colección tomando únicamente en consideración estos aspectos. Sobre todo, porque no puede aplicarse una normativa nacional en un ámbito internacional y, por tanto, en Alemania podría no resultar delito lo que en Perú sí es. Tampoco se podría acudir a la normativa de carácter internacional, donde los hitos más importantes son la Convención Unesco de $1970^{3}$, y el Convenio Unidroit de $1995^{4}$. Principalmente, porque la venta se produjo a finales del siglo XIX, mucho antes de que estos convenios internacionales existiesen, y no pueden aplicarse con carácter retroactivo.

Sería en realidad mucho más efectivo apelar a la opinión pública, al apoyo de la sociedad, o a la propia ética: ¿la compra/venta de esta colección se realizó en términos de igualdad?, ¿dónde debería resguardase una colección como la de María Ana Centeno? Preguntas que tendrán una desigual respuesta, dependiendo del lugar desde el que se lleve a cabo la resolución, enfrentando dos de las clásicas visiones del

3 Medidas que deben adoptarse para prohibir e impedir la importación y la transferencia de propiedad ilícitas de bienes culturales. Disponible en: http://portal.unesco.org/es/ev.php-URL_ ID=13039\&URL_DO=DO_TOPIC\&URL_SECTION=201.html (consultado el 20 de abril de 2020).

4 Convenio Unidroit sobre bienes robados o exportados ilicitamente. Disponible en: http:// www.unesco.org/new/es/culture/themes/illicit-trafficking-of-cultural-property/1995-unidroit-convention/ (consultado el 20 de abril de 2020). 
patrimonio: nacionalismo e internacionalismo cultural (Merryman, 1986). Evidentemente, Perú, un país tradicionalmente emisor de obras de arte, quisiera recuperar gran parte de las obras que se encuentran en el extranjero. Mientras que Alemania se ajustaría a los preceptos del internacionalismo cultural, al poseer un gran número de bienes culturales que abandonaron sus lugares de procedencia en fechas muy similares, siguiendo los preceptos del imperialismo. No debemos olvidar, no obstante, que el Museo estaba actuando según los estándares de su tiempo, y resulta necesario estudiar y analizar los hechos empleando los mismos criterios y formas de pensar que en el momento en que se llevaron a cabo (Kagan, 2013, p. 196).

\section{REFLEXIONES FINALES}

La colección de María Ana Centeno resulta un claro ejemplo de las tendencias adquisitivas y acumulativas de su época: nos muestra cómo una mujer construyó su propio universo a partir y tendencias de su tiempo, de su cultura, y del modo en que ella misma quería ser vista de cara a la sociedad: María Ana coleccionaba las piezas que estaban de moda y eran codiciadas en ese momento, en una actividad que repercutía en su consideración social, y reafirmaba su pertenencia a una élite exclusiva y minoritaria.

El hecho de que esta colección haya sido pensada y realizada por una mujer, debe resultarnos destacable, pues con trabajos como este podemos rescatar y revalorizar su figura. Igualmente, nos permite situarla en un contexto y un momento concretos, teniendo en cuenta que fueron otras muchas las mujeres que decidieron realizar colecciones con presencia de piezas prehispánicas. Entre ellas Astete de Bennet, Hortensia Cáceres Moreno -hermana de la conocida Zoyla Aurora Cáceres Moreno, alias "Evangelina"-, Aline Chazal, o la artista Elena Izcue. Sus colecciones son todas dignas de estudio, al igual que las de sus coetáneos masculinos -mucho más presentes en la historiografía-, pues muestran formas de representación social, y reflejan el modo en que en su momento se valoraban las antigüedades prehispánicas.

Para los límites de nuestro estudio, la colección es destacable porque contiene un importante número de piezas precolombinas. Estas fueron en buena parte a parar al Museo Etnológico de Berlín mediante la compra a los hijos de María Ana, Adolfo y Eduardo Romainville Centeno. Se trata de una transacción que no nos resulta de ningún modo sorprendente, pues formaba parte de la tónica habitual de la época, según la cual los espacios o países considerados "superiores", acudían a naciones como Perú para aumentar sus colecciones. No obstante, sí debemos tener en cuenta que la venta de la colección Centeno ha supuesto una importante pérdida patrimonial para su lugar de origen. Lo cual no necesariamente significa que la colección deba regresar a Perú en su totalidad, pues ante todo defendemos que se lleve a cabo una restitución de tipo moral: el Museo Etnológico de Berlín debería explicar en su discurso expo- 
sitivo la historia de esta colección, y reconocer su ascendencia peruana y el modo en que fue adquirida.

Con este artículo, pretendemos aportar un granito de arena al conocimiento del gabinete de curiosidades de María Ana Centeno, que debe ser considerado, entre otros muchos aspectos, por un temprano interés por las antigüedades prehispánicas. Del mismo modo que nuestra intención ha sido la de situarla en un contexto determinado, en el que los viajeros extranjeros aumentaron su interés por el país y el estudio y adquisición de sus antigüedades precolombinas. Con todo ello, podemos entender mucho mejor las circunstancias que la llevaron a viajar hacia Berlín, y nos resulta mucho más sencillo reflexionar sobre su actual pertenencia y lugar en el mundo. La colección Centeno es peruana, aunque en parte también podríamos considerarla ya alemana, por su relación con el país que la ha cuidado desde finales del siglo XIX. Lo ideal sería que ambas naciones pudieran escribir juntas la historia de esta colección, dando un primer paso hacia la "custodia compartida" del patrimonio, un concepto que debería fomentarse para evitar muchas de las grandes disputas sobre el patrimonio.

\section{BIBLIOGRAFÍA}

Armas Asín, Fernando (2019). Una historia del turismo en el Perú. El Estado, los visitantes y los empresarios (1800-2000). Lima: Fondo Editorial Universidad San Martín de Porres.

Ávalos de Matos, Rosalía y Ravines, Rogger (1974). Las antigüedades peruanas y su protección legal. Revista del Museo Nacional, tomo 40, pp. 363-458.

Bruhns, Karen O. y Kelker, Nancy, L. (2010). Faking the Ancient Andes. Left Coast Press, Inc.

Cabello Carro, Paz (1989). Coleccionismo americano indígena en la España del siglo XVIII. Madrid: Cultura Hispánica.

Catálogo en línea, Museo Etnológico de Berlín. Disponible en: http://www.smb-digital. de/eMuseumPlus?service $=$ ExternalInterface $\&$ module $=$ collection\&moduleFunction=highlight\&filterName=filter.collection.highlights.1300 (consultado en abril de 2020).

Fischer, Manuela (2015). The Inca Collections at the Ethnologisches Museum Berlin. Genesis and contexts. En Tribus. Perspectives on the Inca. Stuttgart: Linden-Museum Stuttgart, Staatliches Museum fur Völkerkunde, pp. 24-37.

Gänger, Stefanie (2014a). The Many Natures of Antiquities: Ana María Centeno and Her Cabinet of Curiosities, Peru, ca. 1832-1874. En Kohk, Philip O., Podgorny, Irina y Gänger, Stefanie (eds.). Natura and Antiquities. The Making of Archaeology in the Americas. University of Arizona Press, pp. 110-124. Disponible en: https://www. jstor.org/stable/j.ctt183gz2c.9?seq=1\#metadata_info_tab_contents 
Gänger, Stefanie (2014b). The Mascapaycha. Collections of Incan Antiquities in Cuzco. En Relics of the Past: The Collecting and Study of Pre-Columbian Antiquities in Peru and Chile, 1837-1911. Oxford: Oxford University Press, pp. 1-92.

García y García, Elvira (1925). La mujer peruana a través de los siglos. Serie historiada de estudios y observaciones. Segundo Tomo. Lima: Imp. Americana-Plazuela del Teatro. Disponible en: https://ufdc.ufl.edu/AA00019316/00002

Glave, Luis Miguel (1986). Agricultura y capitalismo en la tierra sur del Perú (finales del siglo XIX y comienzos del XX). En Deler, Jean-Paul y Saint-Geours, Yves. Estados y naciones en los Andes. Hacia una historia comparativa: Bolivia-Colombia-Ecuador-Perú. Institut Français d'Etudes Andines, Instituto de Estudios Peruanos.

Glave, Luis Miguel (2004). La república instalada. Formación y prensa en el Cuzco. 18251939. Institut Français d'Etudes Andines, Instituto de Estudios Peruanos, 2004. Disponible en: https://books.openedition.org/ifea/4872

Gkozgkou, Dimitra (2013). Los amigos del arte: ¿una sociedad de ambiguos intereses?. En Socias Batet, Inmaculada y Gkozgjou, Dimitra (coords.). Nuevas contribuciones en torno al mundo del coleccionismo en los siglos XIX y XX. Gijón: Trea, pp. 100-125.

Guevara Gil, Armando (1997). La contribución de José Lucas Caparó Muñiz a la formación del Museo Arqueológico de la Universidad del Cuzco. BIRA, n. 24, pp. 167-226. Disponible en: http://repositorio.pucp.edu.pe/index/handle/123456789/114236

Gutiérrez Viñuales, Rodrigo (2013). Recuperación prehispanista en la contemporaneidad. Tradición, vanguardia y fortuna crítica. Revista de Historiografía, n. 19, pp. 88-100. Disponible en: https://www.ugr.es/ rgutierr/PDF1/163.pdf

Kagan, Richard (2013). "El Marqués de la Vega-Inclán y el patrimonio artístico español. ¿Protector o expoliador? En Socias Batet, Inmaculada y Gkozgjou, Dimitra (coords.). Nuevas contribuciones en torno al mundo del coleccionismo en los siglos XIX y XX. Gijón: Trea, pp. 194-205.

Kuon Arce, Elizabeth, Gutiérrez Viñuales, Rodrigo, Gutiérrez, Ramón y Viñuales, Graciela María (2009). Cuzco-Buenos Aires. Ruta de intelectualidad americana (19001950). Cuzco-Buenos Aires-Granada: Universidad de San Martín de Porres, Fondo Editorial.

López Rodríguez, José Ramón (1995). Sevilla, el nacimiento de los museos, América y la botánica. En Gascó La Calle, Fernando y Beltrán, José Luis. La Antigüedad como argumento II: Historiografía de arqueología e historia antigua en Andalucía. Sevilla: Scriptorium, pp. 75-97.

Lumbreras, Luis Guillermo (1998). Max Uhle y la tradición de investigación arqueológica en el Perú. Indiana, n. 15, pp. 177-196. DOI: https://doi.org/10.18441/ind. v15i0.177-196

MacGregor, Arthur (2007). Curiosity and Enlightenment. Collectors and Collections from the Sixteenth to Nineteenth Century. New Heaven and London: Yale University Press. 
Majluf, Natalia y Wuffarden, Luis Eduardo (1999). Elena Izcue. El arte precolombino en la vida moderna. Catálogo de exposición. Museo de Arte de Lima, Telefónica del Perú S.A.A.

Matto de Turner, Clorinda (1890). Bocetos a lápiz de americanos célebres. Lima: Imprenta Bacigalupe. Disponible en: http://www.cervantesvirtual.com/obra-visor/bocetos-al-lapiz-de-americanos-celebres-tomo-primero--0/html/ff49ed0e-82b1-11dfacc7-002185ce6064.html

Merryman, John Henry (1986). Two ways of thinking about cultural property. The American Journal of Cultural Property, vol. 80, n. 4, pp. 831-853. DOI: https://doi. org/10.2307/2202065

Mould de Pease, Mariana (2003). Machu Picchu y el Código de Ética de la sociedad de arqueología: una invitación al diálogo intercultural. Lima: Consejo Nacional de Ciencia y Tecnología, CONCYTEC.

Peluffo, Ana (2004). Bajo las alas del ángel de la caridad: indigenismo y beneficencia en el Perú republicano. Revista Iberoamericana, vol. LXX, n. 206, pp. 103-115. DOI: https://doi.org/10.5195/reviberoamer.2004.5586

Ravines, Rogger (1989). Las investigaciones arqueológicas en el Perú: 1860-1988. Boletín de Lima, n. 60, pp. 17-32.

Ravines, Rogger (1996). V. Defensa, conservación y protección del patrimonio arqueológico. Boletín de Lima, n. 105-106, pp. 145-203.

Rénique, José Luis (2015). Imaginar la nación. Viajes en busca del "verdadero Perú” (18811932). Instituto de Estudios Peruanos.

Riviale, Pascal (2015a). Archaeological collections in Peru and their international influence during the nineteenth century. En Fischer, Manuela y Kraus, Michael (eds.). Exploring the archive. Historical photographs from Latin America. The Collection of the Ethnologisches Museum Berlin. Böhlau Verlag Köln Weimar Wien, Staatliche Museeen zu Berlin, pp. 88-107.

Riviale, Pascal (2015b). Los viajeros franceses en busca del Perú Antiguo (1821-1914). Instituto Francés de Estudios Andinos, Fondo Editorial Pontificia Universidad Católica del Perú. Disponible en: https://books.openedition.org/ifea/3568?lang=es

Tantaleán, Henry (2016). Una historia de la arqueología peruana. Lima: EIP, Universidad San Francisco de Quito (Fuentes e Investigaciones para la Historia del Perú).

Vázquez, Óscar E. (2001). Inventing the Art Collection: Patrons, Markets and the State in Nineteenth-Century Spain. University Park Pa: The Pennsylvania State University Press.

Vilela Galván, Javier Alonso y De la Puente, José Carlos (2011). El último secreto de Machu Picchu. Quién es el dueño de la ciudadela de los Incas. Lima: El Comercio.

Yaya, Isabel (2008). Wonders of America. The curiosity cabinet as a site of representation and knowledge. Journal of the History of Collections, vol. 20, n. 2, pp. 173-188. DOI: https://doi.org/10.1093/jhc/fhm038 
